\title{
Updating the orbital ephemeris of Hercules X-1; rate of decay and eccentricity of the orbit
}

\author{
R. Staubert ${ }^{1}$, D. Klochkov ${ }^{1}$, and J. Wilms ${ }^{2,3}$ \\ 1 Institut für Astronomie und Astrophysik, University of Tübingen, Sand 1, 72076 Tübingen, Germany \\ e-mail: staubert@astro.uni-tuebingen.de \\ 2 Dr. Karl Remeis-Sternwarte, Astronomisches Institut der Universität Erlangen-Nürnberg, Sternwartstr. 7, 96049 Bamberg, Germany \\ ${ }^{3}$ Erlangen Center for Astroparticle Physics (ECAP), Erwin-Rommel-Str. 1, 91058 Erlangen, Germany
}

Received 20 January 2009 / Accepted 30 March 2009

\begin{abstract}
We present an update of the orbital ephemeris of the binary X-ray pulsar Her X-1 and determine an improved value for the rate of orbital decay. In addition, we report the first measurement of the orbital eccentricity. We analyzed pulse timing data of Her X-1 from X-ray observations by RXTE (Rossi X-Ray Timing Explorer) and INTEGRAL over the period 1996-2007. Accurate pulse arrival times were determined from solar system bary-centered photon arrival times by generating pulse profiles averaged over appropriately short integration times. Applying pulse phase connection techniques, it was possible to determine sufficiently accurate local ephemeris data for seven observation periods distributed over 12 years. Combining the new local $T_{\pi / 2}$ values with historical values from the literature we update the orbital ephemeris of Her X-1 to $T_{\pi / 2}=$ MJD 46359.871940(6) and $P_{\text {orb }}=1.700167590(2) \mathrm{d}$ and measure a continuous change of the orbital period of $\mathrm{d} P_{\text {orb }} / \mathrm{d} t=-(4.85 \pm 0.13) \times 10^{-11} \mathrm{~s} \mathrm{~s}^{-1}$. For the first time, a value for the eccentricity of the orbit of Her X-1 has been measured: $\epsilon=(4.2 \pm 0.8) \times 10^{-4}$.
\end{abstract}

Key words. stars: binaries: general - stars: neutron - X-rays: general - X-rays: binaries - X-rays: individuals: Her X-1 - ephemerides

\section{Introduction}

Hercules X-1 and Centaurus X-3 were the first two X-ray pulsars discovered by the Uhuru satellite in 1972 (Tananbaum et al. 1972; Giacconi et al. 1973). Since then they have remained among the most studied X-ray sources. Her X-1 is a close binary system consisting of an accreting magnetized neutron star and the stellar companion HZ Her (first suggested by Liller 1972), a main sequence star of spectral type A/F (Crampton 1974). The mass of the optical companion is $\sim 2.2 M_{\odot}$ (Deeter et al. 1981) which places the system in between high and low mass $\mathrm{X}$-ray binaries. The orbital period of the system is $1.700 \mathrm{~d}$. Due to the high inclination of the orbital plane $\left(i \sim 85^{\circ}-88^{\circ}\right)$ the $\mathrm{X}$-ray source is regularly eclipsed by the optical companion for $\sim 5.5 \mathrm{~h}$ once per orbit. The X-ray luminosity of the source is $L_{\mathrm{X}} \sim 2 \times 10^{37} \mathrm{erg} \mathrm{s}^{-1}$ for a distance of $\sim 7 \mathrm{kpc}$ (Reynolds et al. 1997). The spin period of the neutron star, represented by strong $\mathrm{X}$-ray pulsations, is $1.237 \mathrm{~s}$.

The very first Uhuru observations of Her X-1 revealed the presence of a long-term $\sim 35 \mathrm{~d}$ periodicity which manifests mainly through the alternation of so-called on (high X-ray flux) and off (low X-ray flux) states. The $35 \mathrm{~d}$ cycle contains two on states - the main-on ( $\sim 7$ orbital periods) and the short-on ( $\sim 5$ orbital cycles) - separated by $\sim 4-5$ orbital cycles. This periodicity in Her X-1/HZ Her is usually attributed to a counter-orbitally precessing tilted and warped accretion disk, the outer rim of which periodically blocks the line of sight to the X-ray emitting regions on the surface of the neutron star (see, e.g., Gerend \& Boynton 1976; Howarth \& Wilson 1983; Shakura et al. 1999).
Roughly every $\sim 5$ yrs Her X-1 exhibits so-called anomalous low states where the $\mathrm{X}$-ray flux is strongly reduced and the $35 \mathrm{~d}$ variability is seen only marginally (Parmar et al. 1985; Vrtilek et al. 1994; Parmar et al. 1999; Staubert et al. 2009).

Here we report the timing analysis of X-ray data obtained with RXTE (the Rossi X-ray Timing Explorer) and with INTEGRAL. Combining these results with historical data from the literature we refine the global orbital ephemeris of Her X-1 and rate of the secular decrease of its orbital period, which was originally reported by Deeter et al. (1991). In addition, we were able to measure the eccentricity of the orbit for the first time (only upper limits had been reported before).

\section{Observations}

Her X-1 was repeatedly observed by the Rossi X-ray Timing Explorer (RXTE) (Bradt et al. 1993) over a 10 year time frame (1996-2005). In addition, we make use of two extended observations by INTEGRAL (Winkler et al. 2003) in 2005 and 2007. The observations provided high-quality X-ray spectral and timing data. For the timing analysis presented here we used data obtained with the RXTE Proportional Counter Array (PCA) (Jahoda et al. 1996) in the energy range 3-20 keV and with INTEGRAL ISGRI (Ubertini et al. 2003) in the range 20$60 \mathrm{keV}$. Seven sets of data, all taken during a main on state, were long enough and sufficiently densely sampled such that a local ephemeris could be determined (the set of 2005 is from partly simultaneous observations by RXTE and INTEGRAL, see also Klochkov 2007; Klochkov et al. 2008a,b). We note that the 
Table 1. Details of RXTE observations of Her X-1 used for the timing analysis.

\begin{tabular}{llllllll}
\hline \hline 35 d cycle No. ${ }^{1}$ & 257 & 269 & 303 & 313 & 323 & 351 & 373 \\
\hline Source of observational data & RXTE & RXTE & RXTE & RXTE & RXTE & RXTE/INTEGRAL & INTEGRAL \\
Date of observation & July 96 & Sep. 97 & Dec. 00 & Dec. 01 & Nov. 02 & July 05 & Sep. 07 \\
Start of observation [MJD] & 50290.027 & 50704.912 & 51896.319 & 52243.060 & 52600.061 & 53574.715 & 54346.088 \\
No. of RXTE/INTEGRAL orbits used & 18 & 14 & 33 & 45 & 29 & $8 / 3$ & 1 \\
Elapsed time of observation [days] & 1.42 & 2.43 & 5.62 & 3.52 & 4.02 & 8.16 & 2.03 \\
Elapsed time of obs. [binary orbits] & 0.83 & 1.43 & 3.30 & 2.07 & 2.36 & 4.80 & 1.20 \\
Coverage of binary orbits [\%] & 36.6 & 10.0 & 10.0 & 30.7 & 18.9 & 37.0 & 53.0 \\
Number pulse profiles & 139 & 45 & 61 & 161 & 133 & $9 / 69$ & 27 \\
Mean integration time per profile [s] & 322 & 276 & 802 & 580 & 493 & $420 / 2350$ & 3500 \\
\hline
\end{tabular}

${ }^{1}$ The numbering of $35 \mathrm{~d}$ cycles follows Staubert et al. (1983): "Pulse profile counting" is applied, based on pulse profile shapes repeating with an average period of $34.98 \mathrm{~d}$ (Staubert et al. 2009).

capabilities of relative and absolute timing required for the kind of analysis presented here are well met by both $R X T E$ (Rots et al. 2004) and INTEGRAL (Walter et al. 2003).

Details of the observations are given in Table 1. Following the $35 \mathrm{~d}$ cycle counting method adopted by Staubert et al. (1983) and Staubert et al. (2009) the seven cycles are numbered 257, 269, 303, 313, 323, 351 and 373. Here "pulse profile counting" is applied, as defined by Staubert et al. (2009) (meaning that the changing shape of the pulse profile is used to identify individual 35 d cycles). The PCA data were mostly taken in "event mode", providing arrival times for individual events, and a few times in "binned mode", giving light curves (with time resolution, generally $1 / 16 \mathrm{~s}$, sufficient to perform a timing analysis for the $1.24 \mathrm{~s}$ pulsation in Her X-1).

\section{Timing analysis of RXTE and INTEGRAL data}

\subsection{Basic techniques to determine the pulse period}

We use two techniques for the determination of pulse periods: epoch folding with $\chi^{2}$ search (Holt et al. 1976; Leahy et al. 1983) and pulse phase connection (Manchester \& Taylor 1977; Deeter et al. 1981). Both methods are generally well established and are appropriate in the case where the pulse period is already roughly known. Our data are either "event lists" (the arrival times of single detector events) or "light curves" (containing the numbers of events per second and the absolute times of the centers of those bins).

With epoch folding, events are accumulated into bins of a phase histogram according to their phase with respect to an assumed trial period and a given start time (e.g., the time of the first event), thereby generating a pulse profile (with our Her X-1 data we generally used 128 bins, e.g., Fig. 1). A search for the likely "best" period is conducted by constructing a $\chi^{2}$ distribution: for any pulse profile generated with an assumed trial period the value $\chi^{2}=\Sigma\left(n_{i}-\langle n\rangle\right)^{2} / \sigma_{i}^{2}$ is computed, where $n_{i}$ are the number of events in bin $i$ and $\langle n\rangle$ is the mean of all $n_{i} \cdot \chi^{2}$ tests the deviation from a uniform distribution (which is expected for a trial period far from the true one). The $\chi^{2}$ distribution is found by plotting the $\chi^{2}$ values for a series of trial periods (selected to sample a sufficiently large period range with sufficient resolution). The "best" period is assumed to be the one leading to the pulse profile with the maximum deviation from a flat distribution. The $\chi^{2}$ distribution resembles a triangular distribution and can often be well fitted by a Gaussian, the mean of which may be considered the "best" period (note that this is likely better than using the maximum of the $\chi^{2}$ distribution, especially in cases of limited statistics). The full width at half maximum $(F W H M)$ of the $\chi^{2}$ distribution should be of the order $F W H M \sim P^{2} / T O B$, if $P$ is the true pulse period and TOB is the total elapsed time of the observation.

For pulse phase connection pulse profiles are produced, using the best estimate for the pulse period (e.g., from a $\chi^{2}$ search), for integration intervals of appropriate length (leading to profiles with sufficient statistics). The time differences between profiles of adjacent integration intervals (measured either by making use of sharp features in the profile or by template fitting) are then identified with an integer number of pulse cycles. In this way, a list of unique cycle numbers and associated absolute times are generated, which can then be modeled. Necessary conditions for the applicability of this method are: (1) the shape of the pulse profile must be stable enough for the common details of different pulse profiles to be identified and (2) the time separations of the profiles must be small enough such that, with the given uncertainty of the assumed pulse period, any mis-counting can be ruled out. If these conditions are satisfied the pulse phase connection is by far the preferred technique, allowing very flexible modeling, especially if the pulse period is time variable, and with easy and accurate estimates of uncertainties.

For a constant pulse (spin) period $P_{\mathrm{s}}$ the expected arrival time of pulse number $n$ is

$t_{\mathrm{n}}=t_{0}+n P_{\mathrm{s}}$

where $t_{0}$ is a reference time at the start of the observation. For the case of non-zero first and second derivatives of the pulse period, the arrival times are given by (see, e.g., Kelley et al. 1980; Nagase 1989)

$t_{\mathrm{n}}=t_{0}+n P_{\mathrm{s}}+\frac{1}{2} n^{2} P_{\mathrm{s}} \dot{P}_{\mathrm{s}}+\frac{1}{6} n^{3} P_{\mathrm{s}}^{2} \ddot{P}_{\mathrm{s}}+\ldots$

Here $P_{\mathrm{s}}$ is the initial pulse period valid at $t_{0}$. Modifications for pulse arrival time delays, e.g. because of binary motion of the pulsar, can easily be introduced (see below). Often Eq. (2) is expressed in the inverted form giving the pulse phase $\phi$ at time $\left(t-t_{0}\right)$ (Blandford \& Teukolsky 1976; Manchester \& Taylor 1977; Kramer 2008)

$\phi=\phi_{0}+v_{0}\left(t-t_{0}\right)+\frac{1}{2} \dot{v}\left(t-t_{0}\right)^{2}+\frac{1}{6} \ddot{v}\left(t-t_{0}\right)^{3}+\ldots$

with $v_{0}=1 / P_{\mathrm{s}}$ being the spin frequency at the reference time $t_{0}$. 


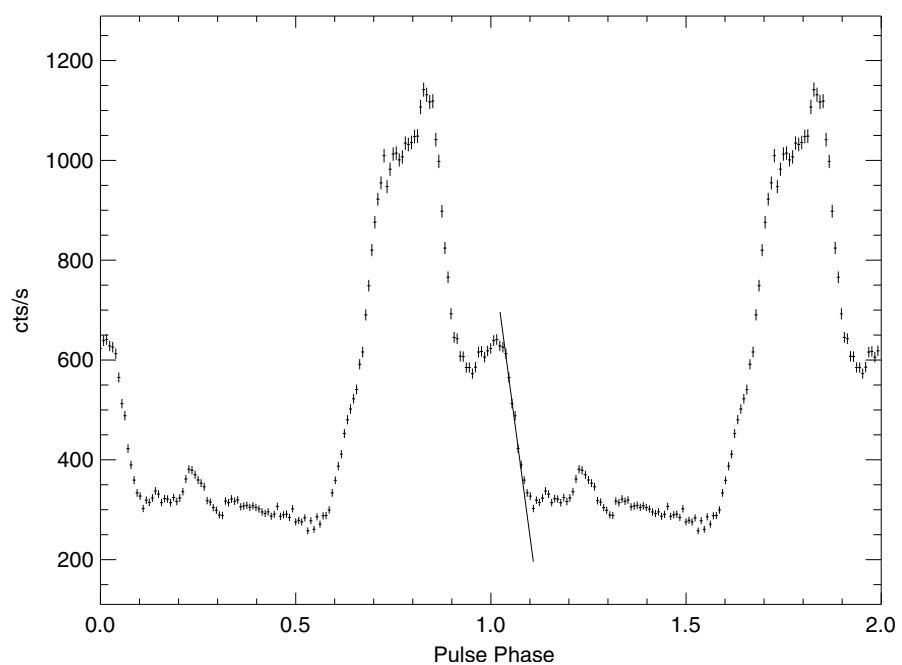

Fig. 1. An example of PCA RXTE pulse profiles used to study pulse shifts due to the orbital motion of Her X-1. The profile is from $700 \mathrm{~s}$ integration time at MJD 52243.786 (35 d cycle No. 313). The solid line shows the sharp decay after the soft trailing shoulder which was used as a phase reference (see text).

\subsection{Analysis with previous ephemeris}

Our initial approach to the timing data of Her X-1 obtained with $R X T E$ was to determine accurate local pulse periods and to construct the corresponding pulse profiles. The scientific motivation was two-fold: (1) to complete our data base on the evolution of the pulse period with time which shows a long-term mean spinup of about $10 \mathrm{~ns}$ per day. Superimposed on this trend, modulations are seen of relative spin-up and spin-down on different time scales, some of which appear to be quasi-periodic and correlated with the turn-on history of the $35 \mathrm{~d}$ modulation due to the precessing accretion disk, e.g., Staubert et al. (2006, 2009); (2) with the spin periods found in this way, pulse profiles were constructed in order to study the systematic variations in pulse shape with phase of the $35 \mathrm{~d}$ modulation. This study has in fact led support for the idea of two different but linked $35 \mathrm{~d}$ clocks in Her X-1: the precession of the accretion disk and free precession of the neutron star (Staubert et al. 2009). Further investigations with regard to the above two points are in progress.

As a first step, all arrival times are translated to the inertial frame of the solar system barycenter. Then, the arrival times are corrected for the binary motion of the X-ray emitting neutron star around its optical companion, using the orbital elements and ephemeris of Her X-1 given by Deeter et al. (1991): $T_{\pi / 2}=$ MJED $43804.519980(14), P_{\text {orb }}=1.700167720(10) \mathrm{d}$, $\dot{P}_{\text {orb }}=(-2.25 \pm 0.27) \times 10^{-8} \mathrm{~d} \mathrm{yr}^{-1}, a \sin i=13.1831(3) \mathrm{s}$, and eccentricity $\epsilon=0.0$. Next, integration intervals are selected, generally identical to the intervals of un-interupted observations (for RXTE the satellite orbits, for INTEGRAL the socalled Science Windows), for which the local pulse period is determined by an epoch folding $/ \chi^{2}$ search and the pulse profile is constructed. A typical profile is shown in Fig. 1. Next, the phase connection technique is applied to all profiles of the groups of observations (Main-On states of Her X-1). Table 1 gives details for the 7 Main-On states covered. This means that within each group each profile is associated with a unique pulse number and an absolute arrival time (in MJD). To find the latter we used a particular feature in the pulse profile of Her X-1 as a time marker, namely the "sharp edge" at the trailing edge of the right hand shoulder of the main pulse going into a flat bottom minimum. This flat bottom is generally well defined, extending over $\sim 0.1$ in pulse phase over which the flux can be represented by a constant (for the example profile shown in Fig. 1 it is from phase $\sim 1.1$ to $\sim 1.2$ ). A straight line is fitted to the "sharp edge" and the phase of the intersection between the straight line and the constant representing the minimum is determined. Since the "sharp edge" is only a few degrees from the vertical the systematic uncertainty introduced by this procedure is quite small. The overall accuracy has been found to be better than one bin for 128 bins per pulse period, that is $<10 \mathrm{~ms}$. Combining this with the known absolute time for phase 0.0 , i.e. the reference time at which the folding of the profile was started, generally the time of the first event, gives the arrival time assigned to this profile. We have selected the "sharp edge" as the time marker, because it appears to be the most stable feature of the pulse profile of Her X-1, while most other features (including the main peak) change their shape as a function of $35 \mathrm{~d}$ phase. We note, however, that the timing results do not depend in any critical way on using the "sharp edge"; consistent results have been obtained on the same data with template fitting using the complete pulse profile (Klochkov 2007; Klochkov et al. 2008b). The data sets used are short enough such that the shape of the pulse profile does not change significantly. It is, of course, necessary that the accuracy of the assumed pulse period is sufficiently high such that no counting errors occur when bridging the existing gaps between data sets.

The difference in energy range in which the data have been taken by RXTE (3-20 keV) and by INTEGRAL (20-60 keV) does not pose a problem for the current timing analysis, nor for the overlapping observations of RXTE and INTEGRAL of 2005 July. First, each data set was analyzed independently, leading to independent, but consistent solutions for the local $T_{\pi / 2}$ values. Since it is known that the pulse profiles of Her X-1 (and other pulsars) change with energy, one might expect that the absolute time of the "sharp edges" is energy dependent. Instead, we have found that the 2005 data sets of both satellites could be combined into a common set. Any shift between the two sub-sets is less than five milli-second (well within the general uncertainty), supporting the notion that the "sharp edge" is indeed a stable feature.

Applying Eq. (2), the observed pulse number/arrival time data were fitted and the best fit values for $t_{0}, P_{\mathrm{s}}$ and $\dot{P}_{\mathrm{s}}$ determined (with $\ddot{P}_{\mathrm{s}} \equiv 0$ ). A first set of $T_{\pi / 2}$ values was obtained which were combined with historical $T_{\pi / 2}$ values from the literature to establish a revised global solution from which updated values for $P_{\text {orb }}$ and $\dot{P}_{\text {orb }}$ for any given time could be calculated. However, we found that systematic sine-like residuals remained with an apparent period close to the orbital period, suggesting that the ephemeris which we had used to correct the arrival times for binary motion was not optimal. As an example, Fig. 2b shows the observed residuals for 35 d cycle No. 313 (Dec. 2001). We therefore decided to perform a timing analysis (starting with barycenter-corrected, but not binary-corrected arrival times) and to determine the local ephemeris for all of our data sets.

\subsection{Analysis of non-binary corrected data}

In the same way as described in Sect. 3.2 the barycenter (not binary) corrected arrival times are used to generate sets of pulse profiles and the corresponding lists containing pairs of pulse numbers and absolute arrival times. These lists were then taken for fits with Eq. (2) which was extended by the additional term

$+a \sin i \cos \left[2 \pi\left(t-T_{\pi / 2}\right) / P_{\text {orb }}\right]$ 

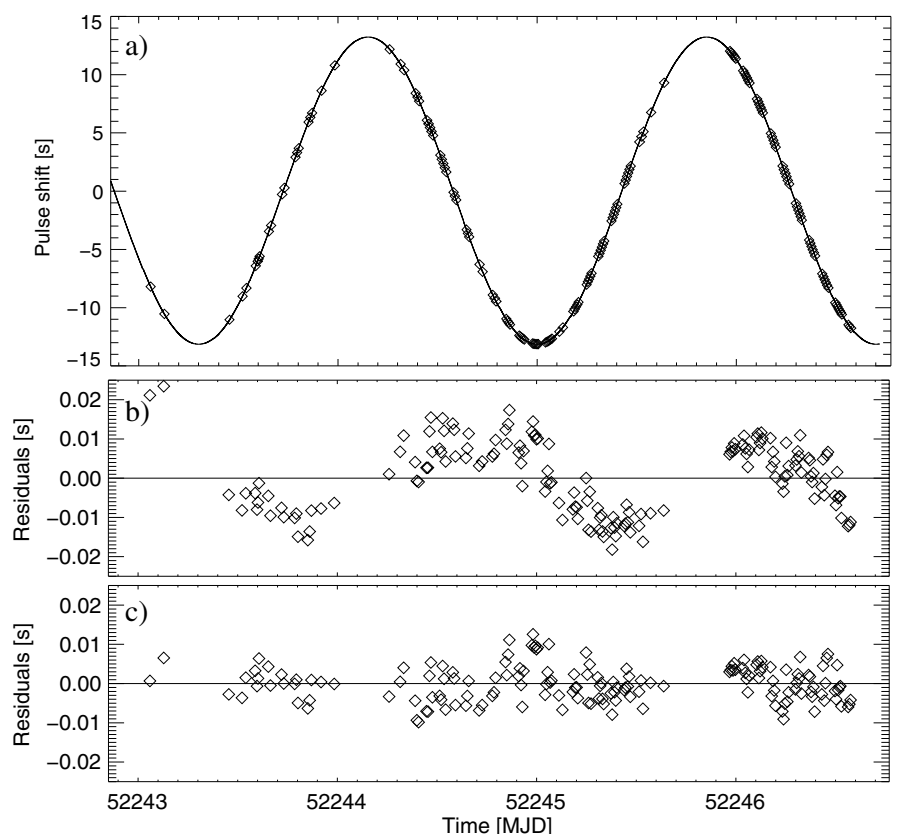

Fig. 2. a) Delays of the pulse arrival time in Her X-1 due to its orbital motion and theoretical sine curve for $35 \mathrm{~d}$ cycle No. 313 (Dec. 2001). b) Residuals to a fit using the orbital parameters based on Deeter et al. (1991). c) Residuals using the best-fit parameters for our analysis of non-binary corrected data.

in order to describe the arrival time delays due to the changing position of the X-ray source along the binary orbit (assumed to be circular). $a \sin i$ is the projected radius of the orbit in light-seconds and $T_{\pi / 2}\left(=T_{90}\right)$ in MJD is the time at which the mean orbital longitude of the neutron star is $90^{\circ}$, corresponding to the maximum delay in pulse arrival time, or zero pulse period derivative (in Her X-1 essentially identical to the center of eclipse). For these fits $a \sin i=13.1831 \mathrm{~s}$ (Deeter et al. 1981, 1991) and orbital periods $P_{\text {orb }}$ (determined for each data set from the global solution found earlier) were kept fixed. The fit parameters, determined for all data sets, are $T_{\pi / 2}, t_{0}, P_{\mathrm{s}}, \dot{P}_{\mathrm{s}}\left(\ddot{P}_{\mathrm{s}} \equiv 0\right)$. For the data set of $35 \mathrm{~d}$ cycle No. 313 (Dec. 2001), the improvement is evident in Fig. 2c).

\subsection{Final fits solving Kepler's equation}

The results obtained so far allow us to establish a global solution with quite accurate values for $P_{\text {orb }}$ and $\dot{P}_{\text {orb }}$ for any time, including the times for all of our observations. Such a procedure is necessary since $P_{\text {orb }}$ cannot be constrained from the individual data sets themselves.

Keeping $P_{\text {orb }}$ fixed at values calculated from the above global solution, we have attempted to find a full orbital solution for each of our individual data sets by solving Kepler's equation (Blandford \& Teukolsky 1976; Nagase 1989), in order to see whether we can constrain other parameters which were so far kept constant: the projected orbital radius $a \sin i$, the eccentricity $\epsilon$, the longitude of periastron passage $\omega$ (in case $\epsilon$ is not zero) and the second derivative of the pulse period $\ddot{P}_{\text {s. }}$.

The first parameter tested was $a \sin i$. Our best data set (cycle No. 313, Dec. 2001) gives $a \sin i=13.1831(5) \mathrm{s}$. For all other data sets the values are consistent with this (within uncertainties). A weighted average of all values leads to $a \sin i=13.1831(4) \mathrm{s}$. The same value was found by

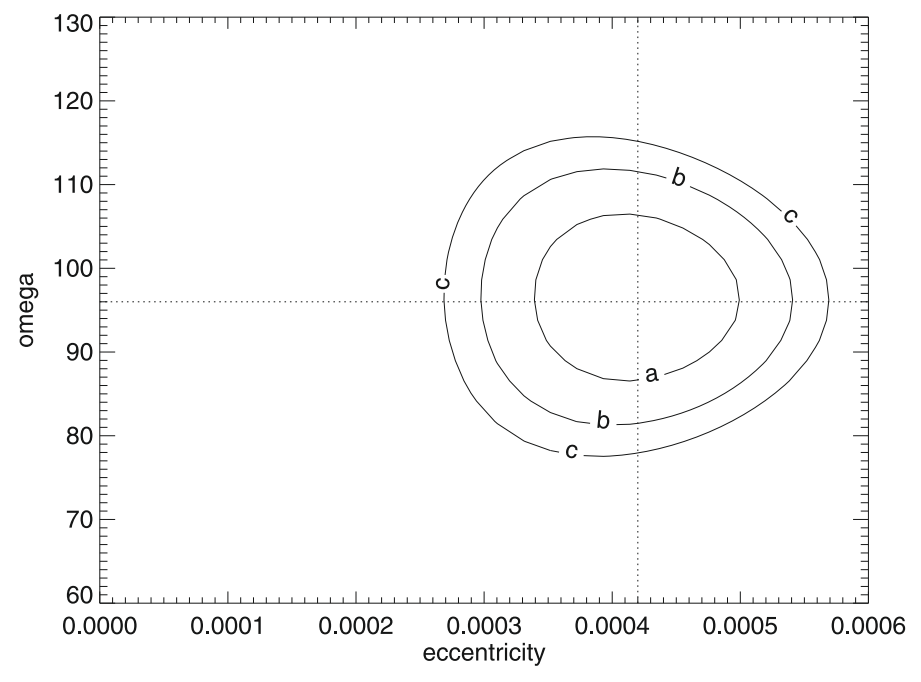

Fig. 3. Uncertainty contours for eccentricity versus omega from fits to the observations of $35 \mathrm{~d}$ cycle No. 313. The error contours are for a: $\chi_{\min }^{2}+1.0, \mathrm{~b}: \chi_{\min }^{2}+2.3$, and $\mathrm{c}: \chi_{\min }^{2}+3.5$.

Deeter et al. (1981, 1991): $a \sin i=13.1831(3)$ s (also a weighted average of several sets of observations). So we have used this value of $a \sin i$ as a constant input parameter. We note here that we had wondered about our inability to reduce the uncertainty of this value, despite the fact that the number of photons we collected from Her X-1 is about 20 times larger than that collected by Deeter et al. (1981). We suspect that this may be due to the intrinsic variability of the pulse profile which introduces additional scattering in pulse arrival times, limiting the achievable accuracy independent of photon statistics.

The second parameter tested was the eccentricity $\epsilon$. To our surprise, the fits of three data sets (35 d cycle Nos. 257, 313 and 323 ) yielded formal values for $\epsilon$ larger than zero. For our best data set (cycle No. 313) a statistically significant value is found: $\epsilon=(4.2 \pm 0.8) \times 10^{-4}$; the corresponding value for the longitude of periastron is $\omega=(96 \pm 10) \mathrm{deg}\left(\chi_{\min }^{2}+1.0\right.$, innermost contour of Fig. 3). This result suggests a significance of slightly more than 5 sigma. In order to further investigate the statistical situation, we applied an F-test (Bevington 1969): the reduction in $\chi^{2}$ when adding $\epsilon$ and $\omega$ as additional free parameters is 27.2 at the final level of $\chi_{2}^{2}=156.6$ with 156 degrees of freedom (d.o.f.). This leads to $F_{\chi}=\left(\Delta \chi^{2} / \chi^{2}\right) /(\Delta$ (d.o.f. $) /$ d.o.f. $)=13.5$, corresponding to a probability of $<10^{-4}$ that the improvement in $\chi^{2}$ is simply due to statistical fluctuations. Further confidence in a significantly measured eccentricity is gained by combining data sets. We combined the data sets of cycle Nos. 313 and 323 into one common set by correcting the arrival times for the local $\dot{P}_{\mathrm{s}}$ values and shifting the arrival times of cycle 323 to the absolute time frame of cycle 313, using our best information on $P_{\text {orb }}$ and $\dot{P}_{\text {orb }}$. The fit to the combined data set yields $\epsilon=(3.8 \pm 0.8) \times 10^{-4}$ and $\omega=(82 \pm 10) \mathrm{deg}$, consistent (within uncertainties) with the results from cycle 313 alone. Adding the data from cycle 257 or cycle 304 again leads to consistent results, but no further improvement. Even though the combination of several data sets confirms and strengthens the results of the single data sets, we prefer to quote the results from cycle 313 alone as our measurement, since it results from the simplest and most straightforward analysis of our best data set. From here onward we have kept $\epsilon$ and $\omega$ fixed at the values measured for cycle 313 .

Finally, we attempted to measure values for the second derivative $\ddot{P}_{\mathrm{s}}$ of the pulse period $P_{\mathrm{s}}$. Only for one data set (cycle 
Table 2. Orbital parameters of Her X-1 determined from the RXTE observations during $35 \mathrm{~d}$ cycles Nos. 257, 269, 303, 313, 323, 351 and from INTEGRAL observations during cycles 351 and $373^{1}$.

\begin{tabular}{llllll}
\hline \hline 35 cycle No. $^{1}$ & $P_{\mathrm{s}}[\mathrm{s}]$ & $T_{\pi / 2}[\mathrm{MJD}(\mathrm{TDB})]$ & $P_{\text {orb }}[\mathrm{d}]$ & $\mathrm{d} P_{\mathrm{s}} / \mathrm{d} t\left[\mathrm{~s} \mathrm{~s}^{-1}\right]$ & $\mathrm{d} P_{\mathrm{s}} / \mathrm{d} t / \mathrm{d} t\left[\mathrm{~s}^{-1}\right]$ \\
\hline 257 & $1.237739612(25)^{2}$ & $50290.659199(20)^{2}$ & 1.700167399 & $-(2.8 \pm 1.0) 10^{-12}$ & 0.0 \\
269 & $1.237729884(35)$ & $50703.799869(50)$ & 1.700167379 & $-(6.8 \pm 2.5) 10^{-12}$ & 0.0 \\
303 & $1.237769831(12)$ & $51895.617167(25)$ & 1.700167321 & $+(1.54 \pm 0.17) 10^{-12}$ & 0.0 \\
313 & $1.237767113(6)$ & $52242.451312(10)$ & 1.700167304 & $-(5.7 \pm 1.5) 10^{-13}$ & 0.0 \\
323 & $1.237761690(4)$ & $52599.486440(12)$ & 1.700167287 & $+(2.8 \pm 0.2) 10^{-12}$ & $-(2.9 \pm 0.08) 10^{-17}$ \\
351 & $1.237758409(8)$ & $53573.682256(35)$ & 1.700167240 & $-(7.0 \pm 0.8) 10^{-13}$ & 0.0 \\
373 & $1.237744750(60)$ & $54345.558195(80)$ & 1.700167202 & 0.0 & 0.0 \\
\hline
\end{tabular}

${ }^{1}$ The numbering of $35 \mathrm{~d}$ cycles follows Staubert et al. (1983): "Pulse profile counting" is applied, based on pulse profile shapes repeating with an average period of $34.98 \mathrm{~d}$ (Staubert et al. 2009). ${ }^{2}$ The uncertainties in parentheses are 1 -sigma $(68 \%)\left(\chi_{\min }^{2}+1.0\right)$ and refer to the last digit(s).

323) is a statistically significant value found; for all others $\ddot{P}_{\text {s }}$ is consistent with zero, for cycle 373 also $\dot{P}_{\mathrm{s}}$ is consistent with zero (see Table 2).

All data sets were then finally fitted, solving Kepler's equation and leaving the following parameters to be determined: $P_{\mathrm{s}}$, $T_{\pi / 2}, t_{0}, \dot{P}_{\mathrm{s}}$ and $\ddot{P}_{\mathrm{s}}$. The final fit parameters and their uncertainties are summarized in Table 2 . The uncertainties quoted are $1 \sigma$ $(68 \%)$, determined from the projections of the $\left(\chi_{\min }^{2}+1.0\right)$ error contours onto the parameter axis of interest, allowing all other interesting parameters to vary during the error contour evaluation (Lampton et al. 1976). The following orbital elements were kept constant: $a \sin i=13.1831 \mathrm{~s}$, omega $=96.0 \mathrm{deg}$ and $\epsilon=(4.2 \pm 0.8) \times 10^{-4}$. The values for $P_{\text {orb }}$ are calculated using the best-fit orbital elements given in Table 3 .

The values of $T_{\pi / 2}$ are significantly different from those calculated using the global solution of Deeter et al. (1991). This is not surprising, given the extrapolation over $\sim 20$ yrs and the updated value for $\dot{P}_{\text {orb }}$. In the next Sect. 4 we will determine the updated global solution for $P_{\text {orb }}$ and $\dot{P}_{\text {orb }}$, using all available historical observations of the source combined with our new results provided in Table 2 .

\section{Orbital ephemeris}

Using our new values of $T_{\pi / 2}$ (Table 2) and the historical data from previous missions (Deeter et al. 1981, 1991; Wilson et al. 1994, and references therein) we determine an improved value for the time derivative of the orbital period $\dot{P}_{\text {orb }}$ and an updated orbital ephemeris of Her X-1 valid for the whole $\sim 36 \mathrm{yrs}$ of observations since the discovery of the source. Modeling all $T_{\pi / 2}$ values (historical observations plus RXTE and INTEGRAL data) with a linear ephemeris $\left(\dot{P}_{\text {orb }} \equiv 0\right)$ results in a very poor fit: $\chi_{\text {red }}^{2}=58.9$ for 28 d.o.f. Thus, the linear ephemeris can clearly be rejected. Figure 4 (upper panel) shows the residuals of $T_{\pi / 2}$ after subtracting the linear part of the quadratic best-fit ephemeris. For the quadratic ephemeris $\left(\dot{P}_{\text {orb }} \neq 0\right.$, shown by the solid curve) the fit gives $\chi_{\text {red }}^{2}=1.8$ for 27 d.o.f. If the outlying data point around MJD 45000 (due to Tenma, Deeter et al. 1991), which deviates from the quadratic fit by more than $3 \sigma$, is removed, $\chi_{\text {red }}^{2}$ is reduced to 1.3 .

The best fit parameters for the quadratic ephemeris are listed in Table 3. $P_{\text {orb }}(0)$ is the orbital period at the reference time $T_{\pi / 2}(0)$. For any particular observation $T_{\pi / 2}$ and $P_{\text {orb }}$ can be found using the following formulae:

$P_{\text {orb }}(t)=P_{\text {orb }}(0)+\dot{P}_{\text {orb }}(0) \cdot\left(t-T_{\pi / 2}(0)\right)$,

$T_{\pi / 2}(n)=T_{\pi / 2}(0)+n P_{\mathrm{orb}}(0)+\frac{1}{2} n^{2} P_{\mathrm{orb}}(0) \cdot \dot{P}_{\mathrm{orb}}(0) ;$

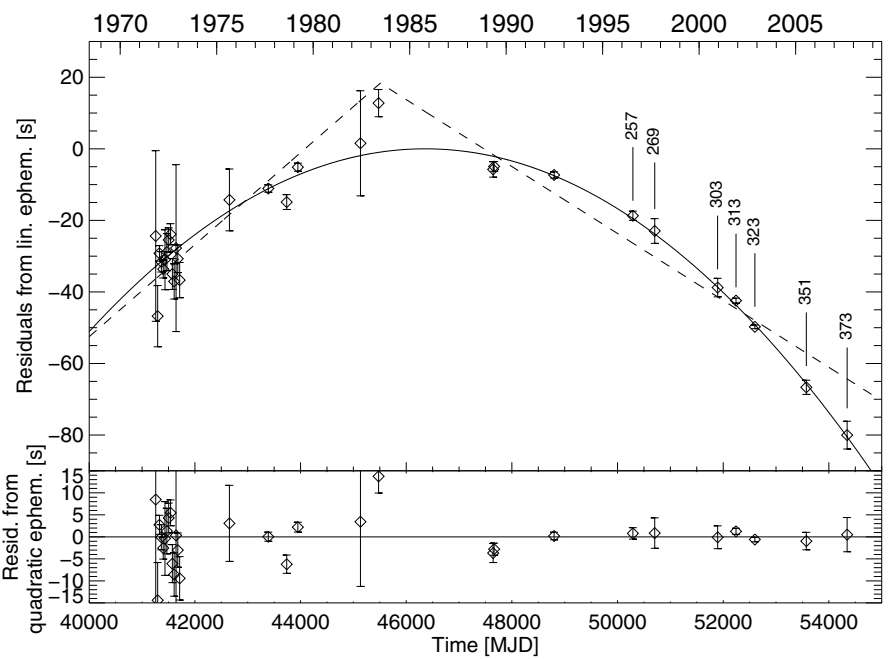

Fig. 4. Upper panel: residuals of $T_{\pi / 2}$ after subtracting the linear part of the quadratic best-fit ephemeris. For the RXTE and INTEGRAL observations analyzed in this work, $35 \mathrm{~d}$ cycle numbers are indicated. The solid line represents the best fit quadratic ephemeris. The dashed lines correspond to fits by the linear ephemeris with a sudden jump of the orbital period around 1983. The lower panel shows the residuals to the best fit quadratic solution.

where $n$ is the orbital cycle number ( $n=0$ corresponds to $\left.T_{\pi / 2}(0)\right)$.

Deeter et al. (1991) had also discussed an alternative model, representing constant orbital periods before and after a sudden change of the orbital period in 1983 (at MJD 45000 ). This appears to be mainly driven by the outlying Tenma data point. We have performed linear fits to the data before and after 1983, they are shown as dashed lines in Fig. 4). While the data set before 1983 is marginally consistent with a linear function $\left(\chi_{\text {red }}=1.8\right.$ for 18 d.o.f.), the data set after 1983 is clearly not: $\chi_{\text {red }}=9.1$ for 8 d.o.f. We, therefore, reject the idea of a sudden change of the orbital period in 1983.

\section{Discussion}

The value of $\dot{P}_{\text {orb }}=-4.85(13) \times 10^{-11} \mathrm{~s} \mathrm{~s}^{-1}$ determined in Sect. 4 and its uncertainty are significantly smaller than the corresponding values determined by Deeter et al. (1991) $(-6.16(74) \times$ $\left.10^{-11}\right)$. In order to connect the derived value of the secular variation of the orbital period with the physics of mass exchange in the system, Deeter et al. (1991) considered a very simplified 
Table 3. Orbital elements of Her X-1.

\begin{tabular}{lll}
\hline \hline$T_{\pi / 2}(0)[\mathrm{MJD}(\mathrm{TDB})]$ & $=46359.871940(6)^{1}$ \\
$P_{\text {orb }}(0)[\mathrm{d}]$ & $=1.700167590(2)$ \\
$\dot{P}_{\text {orb }}(0)[\mathrm{d} / \mathrm{d}]$ & $=$ & $(-4.85 \pm 0.13) \times 10^{-11}$ \\
$a \sin i[1 \mathrm{t}-\mathrm{s}]$ & $=$ & $13.1831(4)^{2}$ \\
eccentricity $\epsilon$ & $=$ & $(4.2 \pm 0.8) \times 10^{-4}$ \\
omega $[\mathrm{deg}]$ & $=96.0 \pm 10.0$ \\
\hline
\end{tabular}

${ }^{1}$ The uncertainties in parentheses (68\%) refer to the last digit(s).

${ }^{2}$ Note that Deeter et al. (1981) had measured $a \sin i=13.1831$ (3) lt-s (with a slightly smaller uncertainty). All other values are updated or new.

model, in which the two stars comprising the binary are treated as point masses and only orbital angular momentum is considered. In the case of conservative mass transfer one can obtain:

$$
\frac{\dot{M}_{\mathrm{ns}}}{M_{\mathrm{ns}}}-\frac{\dot{M}_{\mathrm{ns}}}{M_{\mathrm{opt}}}+\frac{\dot{P}_{\mathrm{orb}}}{3 P_{\mathrm{orb}}}=0,
$$

where $M_{\mathrm{ns}}$ and $M_{\mathrm{opt}}$ are the masses of the neutron star and the optical companion, respectively, and $\dot{M}_{\mathrm{ns}}$ is the accretion rate of the neutron star. In this idealized case, using $M_{\mathrm{ns}}=1.4 M_{\odot}$, $M_{\text {opt }}=2.2 M_{\odot}$, and the value of $\dot{P}_{\text {orb }}=-4.85 \times 10^{-11} \mathrm{~s} / \mathrm{s}$, we obtain a mass accretion rate of the neutron star of $\dot{M}_{\mathrm{ns}} \sim$ $1.3 \times 10^{-8} M_{\odot} \mathrm{yr}^{-1} \sim 8 \times 10^{17} \mathrm{~g} \mathrm{~s}^{-1}$. This value is slightly smaller than that found by Deeter et al. (1981) but still roughly an order of magnitude higher than the accretion rate necessary to provide the observed X-ray luminosity $L_{\mathrm{X}} \sim 2 \times 10^{37} \mathrm{erg} \mathrm{s}^{-1}$ (assuming that the radiative efficiency of accretion is $\sim 10 \%$ ). To explain this, Deeter et al. (1981) suggested that the mass transfer is not conservative $\left(\dot{M}_{\mathrm{ns}} \neq-\dot{M}_{\mathrm{opt}}\right)$ and matter may leave the system in the form of magnetically channeled wind from the X-ray heated surface of the companion star (see Ruderman et al. 1989), carrying away angular momentum. We argue, however, that the model used for conservative mass transfer is very approximate. It does not take into account the angular momentum of the accretion disk and the accretion stream, their dynamic action on the stars as well as the shift of the center of mass of the system during the mass transfer, which are known to be significant factors influencing the final result (see e.g. D'Souza et al. 2006). Furthermore, one might expect exchange of angular momentum by spin-orbit coupling (Marsh et al. 2004). Thus, Eq. (7) is very approximate and may provide only a rough estimate (to the order of magnitude) of $\dot{M}_{\text {ns }}$. The discrepancy we observe, therefore, cannot rule out a conservative mass transfer scenario.

The alternative to a continuous decrease of the orbital period, namely a sudden change of the orbital period around 1983 as discussed by Deeter et al. (1981), has received observational support by the analysis of Stelzer et al. (1997), who performed a fit of the timing data of Her X-1 available at that time, finding that the model with a sudden jump provided a smaller $\chi^{2}$ than the model assuming continuous decrease of $P_{\text {orb }}$. Our current observational result clearly rejects this conclusion (Sect. 4). This is reassuring in the light of several theoretical difficulties which were discussed by Deeter et al. (1981). Here we add that from the disk-stream coupling model described in Staubert et al. (2009) we expect a decrease of the mass transfer to the neutron star during Anomalous Lows (AL), not an increase as assumed by Deeter et al. (1981). This is supported by the observed spindown during most of ALSs (Staubert et al. 2006). Thus, we argue that the decrease of the orbital period of Her X-1/HZ Her originally discovered by Deeter et al. (1981) is due to a constant negative $\dot{P}_{\text {orb }}$ and is not confined to any short time interval (like a jump around 1983) and that the most probable explanation is a continuous mass transfer from HZ Her to the neutron star in a conservative or non-conservative scenario.

\section{Summary and conclusions}

The precise timing analysis of the RXTE and INTEGRAL observations in combination with the historical data allowed us to improve the value for the secular change of the orbital period in Her X-1/HZ Her, originally reported by Deeter et al. (1981). Our absolute value of $\dot{P}_{\text {orb }}$ is somewhat smaller than that determined by Deeter et al. (1981), and the uncertainty is significantly less. We conclude that we cannot rule out the conservative mass transfer scenario. The data rule out the possibility of a sudden jump of the orbital period around 1983 as an alternative for a continuous decrease. The new value for $\dot{P}_{\text {orb }}$ along with the values of $T_{\pi / 2}$ and $P_{\text {orb }}$ (listed in Table 3 ) establish a new orbital ephemeris of Her X-1 that can be used to calculate the orbital period, $P_{\text {orb }}$, and the time $T_{\pi / 2}$ for any particular time.

A further result is the first measurement of a non-zero eccentricity $\epsilon=(4.2 \pm 0.8) \times 10^{-4}$ of the Her X-1/HZ Her orbit. For most practical purposes, such a small deviation from zero may not be relevant. However, in searches for subtle effects, e.g. a precession of the plane of the orbit, possibly associated with precession of HZ Her (Deeter \& Boynton 1976; Deeter et al. 1991), such accurate values may be needed.

Acknowledgements. We acknowledge the support through DFG grants Sta $173 / 31$ and 436 RUS 113/717/0-1 and the corresponding RBFR grants RFFINNIO-03-02-04003 and RFFI 06-02-16025, as well as DLR grant 50 0R 0302. We thank L. Rodina for her endless efforts with respect to the data reduction, $\mathrm{K}$. Postnov for discussions about (non)conservative mass transfer in binaries and $\mathrm{R}$. Rothschild for valuable comments on the manuscript. We thank the anonymous referee for relevant questions and valuable suggestions.

\section{References}

Bevington, P. R. 1969, in Data reduction and Error Analysis for the Physical Sciences (N. Y. Mcgraw-Hill)

Blandford, R., \& Teukolsky. 1976, ApJ, 205, 580

Bradt, H. V., Rothschild, R. E., \& Swank, J. H. 1993, A\&AS, 97, 355

Crampton, D. 1974, ApJ, 187, 345

Deeter, J. E., \& Boynton, P. E. 1976, ApJ, 210, L133

Deeter, J. E., Pravdo, S. H., \& Boynton, P. E. 1981, ApJ, 247, 1003

Deeter, J. E., Boynton, P. E., Miyamoto, S., et al. 1991, ApJ, 383, 324

D'Souza, M. C. R., Motl, P. M., Tohline, J. E., \& Frank, J. 2006, ApJ, 643, 381

Gerend, D., \& Boynton, P. E. 1976, ApJ, 209, 562

Giacconi, R., Gursky, H., Kellogg, E., et al. 1973, ApJ, 184, 227

Holt, S., Boldt, E., Kaluzienski, L., Serlemitsos, P., \& Swank, J. 1976, Nature, 263,484

Howarth, I. D., \& Wilson, B. 1983, MNRAS, 202, 347

Jahoda, K., Swank, J. H., Giles, A. B., et al. 1996, in EUV, X-Ray, and GammaRay Instrumentation for Astronomy VII, ed. O. H. Siegmund, \& M. A. Gummin, Proc. SPIE, 2808, 59

Kelley, R., Rappaport, S., \& Petre, R. 1980, ApJ, 238, 699

Klochkov, D. 2007, Ph.D. Thesis, University of Tübingen, Germany,

http://astro.uni-tuebingen.de/publications/diss.shtml Klochkov, D., Staubert, R., Postnov, K., et al. 2008a, A\&A, 482, 907

Klochkov, D., Staubert, R., Postnov, K., et al. 2008b, in Proc. 7th INTEGRAL

Workshop, Copenhagen, Sep. 2008, ed. N. Lund, \& et al., submitted

Kramer, M. 2008, in Rev. Mod. Astron., ed. S. Röser (Wiley WCH), 255

Lampton, M., Margon, B., \& Bowyer, S. 1976, ApJ, 208, 177

Leahy, D. A., Elsner, R. F., \& Weisskopf, M. C. 1983, ApJ, 272, 256

Liller, W. 1972, IAU Circ., 2415, 1

Manchester, R., \& Taylor, J. 1977, Pulsars (San Francisco: Freeman)

Marsh, T. R., Nelemans, G., \& Steeghs, D. 2004, MNRAS, 350, 113

Nagase, F. 1989, PASJ, 41, 1

Parmar, A. N., Pietsch, W., McKechnie, S., et al. 1985, Nature, 313, 119 
Parmar, A. N., Oosterbroek, T., dal Fiume, D., et al. 1999, A\&A, 350, L5 Reynolds, A. P., Quaintrell, H., Still, M. D., et al. 1997, MNRAS, 288, 43 Rots, A., Jahoda, K., \& Lyne, A. 2004, ApJ, 605, L129

Ruderman, M., Shaham, J., Tavani, M., \& Eichler, D. 1989, ApJ, 343, 292

Shakura, N. I., Prokhorov, M. E., Postnov, K. A., \& Ketsaris, N. A. 1999, A\&A, 348, 917

Staubert, R., Bezler, M., \& Kendziorra, E. 1983, A\&A, 117, 215

Staubert, R., Schandl, S., Klochkov, D., et al. 2006, in The Transient Milky Way: A Perspective for MIRAX, ed. J. Braga, F. D'Amico, \& R. E. Rothschild, AIP Conf. Ser., 840, 65

Staubert, R., Klochkov, D., Postnov, K., et al. 2009, A\&A, 494, 1025
Stelzer, B., Staubert, R., Wilms, J., et al. 1997, in Proceedings of the Fourth Compton Symposium, ed. C. D. Dermer, M. S. Strickman, \& J. D. Kurfess, AIP Conf. Ser., 410, 753

Tananbaum, H., Gursky, H., Kellogg, E. M., et al. 1972, ApJ, 174, L143

Ubertini, P., Lebrun, F., Di Cocco, G., et al. 2003, A\&A, 411, L131

Vrtilek, S. D., Mihara, T., Primini, F. A., et al. 1994, ApJ, 436, L9

Walter, R., Favre, P., Dubath, P., et al. 2003, A\&A, 411, L25

Wilson, R. B., Finger, M. H., Pendleton, G. N., Briggs, M., \& Bildsten, L. 1994, in The Evolution of X-ray Binaries, ed. S. Holt, \& C. S. Day, AIP Conf. Ser., 308,475

Winkler, C., Courvoisier, T. J.-L., Di Cocco, G., et al. 2003, A\&A, 411, L1 